\title{
Analytical model for ultrasonic modulation of multiply scattered light: an extension from isotropic scattering to anisotropic scattering
}

Sava Sakadzic, Lihong V. Wang

Sava Sakadzic, Lihong V. Wang, "Analytical model for ultrasonic modulation of multiply scattered light: an extension from isotropic scattering to anisotropic scattering," Proc. SPIE 4960, Biomedical Optoacoustics IV, (1 July 2003); doi: $10.1117 / 12.477786$

SPIE. Event: Biomedical Optics, 2003, San Jose, CA, United States 


\title{
Analytical model for ultrasonic modulation of multiply scattered light: an extension from isotropic scattering to anisotropic scattering
}

\author{
Sava Sakadžić and Lihong V. Wang \\ Optical Imaging Laboratory, Department of Biomedical Engineering, \\ Texas A\&M University, 3120 TAMU, College Station, Texas 77843-3120, \\ http://oilab.tamu.edu
}

\begin{abstract}
We have calculated analytically the temporal autocorrelation function of the electric field component of multiply scattered coherent light transmitted through an anisotropically scattering mediuma irradiated with a plane ultrasonic wave. The accuracy of the analytical solution is verified with an independent Monte Carlo simulation. The analytical model shows that an approximate similarity relation exists.
\end{abstract}

Keywords: Ultrasonic modulation, multiply scattered light, autocorrelation function, Monte Carlo

\section{INTRODUCTION}

Recently ultrasound-modulated optical tomography has been established as a new and growing area of research. Potential applications exist in the imaging of scattering media, especially biological tissues. This technique combines ultrasonic resolution and optical contrast based on the differences in optical properties among different types of tissues. The collective motion of the scatterers and the periodical changes in the index of refraction that are generated by focused ultrasound produce fluctuations in the intensity of the speckles formed by the multiply scattered light. By measuring the depth of intensity fluctuations, we can spatially localize differences in optical properties inside a scattering medium. Intensive research has been conducted by several groups in the past few years $^{1-12}$ in an attempt to explain the mechanism of ultrasonic tagging of light and to develop practical systems based on this new imaging modality. However, additional work is needed to advance our understanding of this relatively new phenomenon.

In our simple model we will consider two basic mechanisms that are responsible for variation in the optical phase of multiply scattered light. With the first mechanism, the variation in the phase is caused by ultrasoundinduced collective displacements of scatterers, which was modeled for the first time by Leutz and Maret. ${ }^{10}$ With the second mechanism, the variation in the phase is caused by ultrasound-induced variation of the index of refraction, which was modeled, in combination with the first mechanism, by Wang. ${ }^{11,12}$ The current models, however, are based on non-absorbing and isotropic scattering media rather than the more realistic absorbing and anisotropic scattering media.

In this paper we extend the solution for the temporal autocorrelation function of the electrical field component obtained in, ${ }^{11}$ incorporating into the model a general scattering phase function. The organization of the paper is as follows. Section 2 describes the derivation of the autocorrelation function of the ultrasound-modulated electric field along paths of length $s$ while the detailed derivations are deferred to the appendix. In Sect. 3 we incorporate the expressions obtained in Sect. 2 into the solution for the total electric field autocorrelation function transmitted through a scattering slab in the case of a plane source of coherent light and a point detector. We examine the accuracy of our analytical solution with an independent Monte Carlo simulation in Sec. 4. In Sect. 5 we use both the Monte Carlo simulation as well as the analytical solution for the autocorrelation function to explore the validity of the similarity relation. Finally, a brief summary of our conclusions is presented. 


\section{AUTOCORRELATION OF A SINGLE PATHLENGTH}

Consider the propagation of coherent light through a homogeneous scattering medium irradiated by a plane ultrasonic wave. If we neglect all the polarization effects, the temporal autocorrelation function of the electric field component of the scattered light at the point detector position can be written as follows:

$$
G_{1}(\tau)=\left\langle E(t) E^{*}(t+\tau)\right\rangle .
$$

We assume that the photon mean free path is much longer than the optical wavelength (weak scattering) and the acoustic amplitude is much less than the optical wavelength. In this weak scattering approximation, the correlation between different random paths vanishes and only the photons travelling along the same path of length $s$ produce a nonzero effect. Consequently, the autocorrelation function becomes ${ }^{10,11}$

$$
G_{1}(\tau)=\int_{0}^{\infty} p(s)\left\langle E_{s}(t) E_{s}^{*}(t+\tau)\right\rangle_{U}\left\langle E_{s}(t) E_{s}^{*}(t+\tau)\right\rangle_{B} d s
$$

where $p(s)$ is the probability density function of path length $s$. In Eq. (2) we assume that contributions from Brownian motion (B) and ultrasound (U) are independent and that we can separate them.

The remaining task in this section is to consider the ultrasound component of Eq. (2) when photon scattering is anisotropic. Following the derivations in, ${ }^{10,11}$ the autocorrelation for paths of length $s$ can be written as

$$
\left\langle E_{s}(t) E_{s}^{*}(t+\tau)\right\rangle_{U}=\left\langle\exp \left\{-i\left[\sum_{j=1}^{N} \Delta \phi_{n, j}(t, \tau)+\sum_{j=1}^{N-1} \Delta \phi_{d, j}(t, \tau)\right]\right\}\right\rangle .
$$

In Eq. (3), $\Delta \phi_{n, j}(t, \tau)=\phi_{n, j}(t+\tau)-\phi_{n, j}(t)$, where $\phi_{n, j}(t)$ is the phase variation induced by the modulated index of refraction along the $j$ th free path and $\Delta \phi_{d, j}(t, \tau)=\phi_{d, j}(t+\tau)-\phi_{d, j}(t)$, where $\phi_{d, j}(t)$ is the phase variation induced by the modulated displacement of the $j$ th scatterer following the $j$ th free path. Summation is going over all $N$ free paths and $N-1$ scattering events along the photon path. Averaging is over time and over all the photon paths of length $s$. When the phase variation is small (much less than unity), we can approximate Eq. (3) with

$$
\left\langle E_{s}(t) E_{s}^{*}(t+\tau)\right\rangle_{U}=\exp (-F(\tau) / 2),
$$

where the function $F(\tau)$ is

$$
F(\tau)=\left\langle\left[\sum_{j=1}^{N} \Delta \phi_{n, j}(t, \tau)+\sum_{j=1}^{N-1} \Delta \phi_{d, j}(t, \tau)\right]^{2}\right\rangle
$$

Let us assume that the plane ultrasound waves propagate along the $Z$ direction with wave vector $\mathbf{k}_{a}=k_{a} \hat{\mathbf{e}}_{a}$, where ${ }^{\wedge}$ indicates a unity vector, and $k_{a}=2 \pi / \lambda_{a}$, where $\lambda_{a}$ is the ultrasonic wavelength. Along the photon path with $N$ free paths, the positions of the $N-1$ scatterers are $\mathbf{r}_{1}, \mathbf{r}_{2}, \ldots, \mathbf{r}_{N-1}$. We will associate each free path between two consecutive scattering events with a vector $\mathbf{l}_{j}=\mathbf{r}_{j}-\mathbf{r}_{j-1},\left(\mathbf{l}_{j}=l_{j} \hat{\mathbf{e}}_{j}\right)$. The expressions for $\Delta \phi_{n, j}(t, \tau)$ and $\Delta \phi_{d, j}(t, \tau)$ in terms of the ultrasound amplitude $A$, background index of refraction $n_{0}$, and the amplitude of the optical wave vector $k_{0}$ are $^{11}$

$$
\begin{aligned}
\Delta \phi_{n, j}(t, \tau)= & \left(4 n_{0} k_{0} A \eta\right) \sin \left(\omega_{a} \tau / 2\right) \sin \left[(1 / 2) k_{a} l_{j} \cos \theta_{j}\right]\left(\cos \theta_{j}\right)^{-1} \\
& \times \cos \left[\mathbf{k}_{a} \cdot \mathbf{r}_{j-1}+(1 / 2) k_{a} l_{j} \cos \theta_{j}-\omega_{a} t-\omega_{a} \tau / 2\right], \\
\Delta \phi_{d, j}(t, \tau)= & \left(2 n_{0} k_{0} A\right) \sin \left(\omega_{a} \tau / 2\right)\left[\left(\hat{\mathbf{e}}_{j+1}-\hat{\mathbf{e}}_{j}\right) \cdot \hat{\mathbf{e}}_{a}\right] \cos \left(\mathbf{k}_{a} \cdot \mathbf{r}_{j}-\omega_{a} t-\omega_{a} \tau / 2\right),
\end{aligned}
$$


where coefficient $\eta$ depends on the acoustic velocity of the material $v_{a}$, the density of the medium $\rho$, and the adiabatic piezo-optical coefficient $\partial n / \partial p: \eta=(\partial n / \partial p) \rho v_{a}^{2}$. In Eqs. (6) and (7), $\theta_{j}$ is the angle between the propagation directions of the light and ultrasound $\left(\cos \theta_{j}=\hat{\mathbf{e}}_{a} \cdot \hat{\mathbf{e}}_{j}\right)$, and $\omega_{a}=2 \pi f_{a}$, where $f_{a}$ is the ultrasonic frequency.

Now we can express the function $F(\tau)$ from Eq. (5) as

$$
\begin{aligned}
F(\tau)= & \left\langle\sum_{j=1}^{N}\left(\Delta \phi_{n, j}(t, \tau)\right)^{2}\right\rangle_{t, \Pi(s)}+\left\langle 2 \sum_{j=2}^{N} \sum_{k=1}^{j-1} \Delta \phi_{n, j}(t, \tau) \Delta \phi_{n, k}(t, \tau)\right\rangle_{t, \Pi(s)} \\
& +\left\langle\sum_{j=1}^{N-1}\left(\Delta \phi_{d, j}(t, \tau)\right)^{2}\right\rangle_{t, \Pi(s)}+\left\langle 2 \sum_{j=2}^{N-1} \sum_{k=1}^{j-1} \Delta \phi_{d, j}(t, \tau) \Delta \phi_{d, k}(t, \tau)\right\rangle_{t, \Pi(s)} \\
& +\left\langle 2 \sum_{j=1}^{N} \sum_{k=1}^{N-1} \Delta \phi_{n, j}(t, \tau) \Delta \phi_{d, k}(t, \tau)\right\rangle_{t, \Pi(s)} .
\end{aligned}
$$

The averaging over time $t$ of each term on the right side of Eq. (8) is an easy task, while the averaging over all the allowed paths $\Pi(s)$ of length $s$ with $N$ free paths is more difficult. In order to simplify the probability density function of a particular photon path $p\left(\mathbf{l}_{1}, \ldots, \mathbf{l}_{N}\right)$, we will first make some assumptions. The number of steps $N$ in each photon path in the diffusion regime is much larger than unity. Consequently, even if the total path length $s$ is fixed, the correlation between the lengths of free paths $l_{j}$ is still weak. As a result, we have

$$
p\left(\mathbf{l}_{1}, \ldots, \mathbf{l}_{N}\right)=p\left(l_{1}\right) p\left(l_{2}\right) \ldots p\left(l_{N}\right) g\left(\hat{\mathbf{e}}_{1}, \ldots, \hat{\mathbf{e}}_{N}\right),
$$

where $p\left(l_{j}\right)=l^{-1} \exp \left(-l_{j} / l\right)$ is the probability density for a photon to travel a distance $l_{j}$ between two scattering events, and $g\left(\hat{\mathbf{e}}_{1}, \ldots, \hat{\mathbf{e}}_{N}\right)$ is the probability density for the photon to travel along the directions $\hat{\mathbf{e}}_{1}, \ldots, \hat{\mathbf{e}}_{N}$. Because the probability of scattering a photon travelling in direction $\hat{\mathbf{e}}_{j}$ into direction $\hat{\mathbf{e}}_{j+1}$ is described with phase function $f\left(\hat{\mathbf{e}}_{j} \cdot \hat{\mathbf{e}}_{j+1}\right)$, we can write Eq. (9) as

$$
p\left(\mathbf{l}_{1}, \ldots, \mathbf{l}_{N}\right)=p_{s}\left(\hat{\mathbf{e}}_{1}\right) \prod_{j=1}^{N} p\left(l_{j}\right) \prod_{j=1}^{N-1} f\left(\hat{\mathbf{e}}_{j} \cdot \hat{\mathbf{e}}_{j+1}\right),
$$

where $p_{s}\left(\hat{\mathbf{e}}_{1}\right)$ is the probability density function of the starting photon direction $\hat{\mathbf{e}}_{1}$ in the scattering medium. Note that we assumed the phase function does not depend on the azimuth angle or the incident direction. becomes

Using Eq. (10) as the probability density function and going through some algebra (see Appendix), Eq. (8)

$$
F(\tau) \simeq \frac{s}{l}\left(2 n_{0} k_{0} A\right)^{2} \sin ^{2}\left(\frac{1}{2} \omega_{a} \tau\right)\left\{\eta^{2}\left(k_{a} l\right)^{2} \Re\left[\hat{J}(\hat{I}-\hat{J})^{-1}\right]_{0,0}+\frac{1}{3}\left(1-g_{1}\right)\right\},
$$

where $\Re\left[\hat{J}(\hat{I}-\hat{J})^{-1}\right]_{0,0}$ represents the real part of the $(0,0)$ element of the matrix $\hat{J}(\hat{I}-\hat{J})^{-1}$ and the elements of the matrix $\hat{J}$ are defined as

$$
J_{m, n}=g_{m}^{1 / 2} g_{n}^{1 / 2} \sqrt{\frac{2 m+1}{2}} \sqrt{\frac{2 n+1}{2}} \int_{-1}^{1} T(x) P_{m}(x) P_{n}(x) d x, \quad T(x)=\frac{1}{1-i k_{a} l x},
$$

where $P_{j}(x)$ is a Legendre polynomial of order $j$, and $g_{j}$ is the $j$ th Legendre polynomial expansion coefficient of the scattering phase function [Eq. (24)]. Thus, $g_{1}$ is equal to the scattering anisotropy factor $g$, i.e., the average 
cosine of the scattering angle. The value $\Re\left[\hat{J}(\hat{I}-\hat{J})^{-1}\right]_{0,0}$ is the limit of the $\Re\left[\hat{J}_{Q}\left(\hat{I}_{Q}-\hat{J}_{Q}\right)^{-1}\right]_{0,0}$ when $Q$ approaches infinity, where $\hat{J}_{Q}$ is the $Q \times Q$ matrix whose elements are defined by Eq. (12).

We will rearrange the expression for $F(\tau)$ to

$$
F(\tau)=s\left(2 n_{0} k_{0} A\right)^{2} \sin ^{2}\left(\omega_{a} \tau / 2\right)\left(\delta_{n}+\delta_{d}\right)
$$

where

$$
\delta_{n}=\eta^{2} k_{a}^{2} l \Re\left[\hat{J}(\hat{I}-\hat{J})^{-1}\right]_{0,0}, \quad \delta_{d}=(1-g) /(3 l) .
$$

\section{AUTOCORRELATION FOR A SLAB: ANALYTICAL SOLUTION}

In this section, we will test the accuracy of our analytical expression for $F(\tau)$ from the previous section with an independent Monte Carlo simulation in the case of an infinitely wide scattering slab. Slab geometry has been considered previously for various particular problems. ${ }^{10-16}$ We will solve Eq. (2) for anisotropically scattering and absorbing media based on the expression for function $F(\tau)$ obtained in the previous section.

The $Z$ axis of the coordinate system is perpendicular to the infinitely wide slab of thickness $L$. The index of refraction of both the surrounding and scattering media is $n_{0}$. A plane ultrasonic wave propagates along the slab (in the $X-Y$ plane) and is assumed to fill the whole slab. At the same time, one side of the slab is irradiated by a plane electromagnetic wave, and a point detector measures the temporal autocorrelation function of the electric field component on the other side of the slab. By solving the diffusion equation for such geometry, it is possible to find a reasonably good expression ${ }^{11,13,16}$ for the photon path length probability density function $p(s)$. We follow the derivation of $p(s)$ from ${ }^{11,13}$ by applying an infinite number of image sources and introducing extrapolated-boundary conditions ${ }^{13,16}$ to obtain the following expression:

$$
\begin{gathered}
p(s)=\quad K(s) \sum_{i=0}^{\infty}\left\{\left[(2 i+1) L_{0}-z_{0}\right] \exp \left(-\frac{\left[(2 i+1) L_{0}-z_{0}\right]^{2}}{4 D s}\right)\right. \\
\left.-\left[(2 i+1) L_{0}+z_{0}\right] \exp \left(-\frac{\left[(2 i+1) L_{0}+z_{0}\right]^{2}}{4 D s}\right)\right\} \\
K(s)=\frac{1}{2 \sqrt{\pi D}} \frac{\sinh \left(L_{0} \sqrt{\mu_{a} D^{-1}}\right)}{\sinh \left(z_{0} \sqrt{\mu_{a} D^{-1}}\right)} s^{-3 / 2} \exp \left(-\mu_{a} s\right)
\end{gathered}
$$

where $D=l^{*} / 3$ is the diffusion constant; $L_{0}$ is the distance between the two extrapolated boundaries of the slab; $z_{0}$ is the location of the converted isotropic source from the extrapolated incident boundary of the slab; and $l^{*}$ is the isotropic scattering mean free path defined as $l^{*}=l /(1-g)$. The distance between the extrapolated boundary and the corresponding real boundary of the slab is $l^{*} \gamma(\gamma=0.7104)$. The converted isotropic source is one isotropic scattering mean free path into the slab. Therefore, $L_{0}=L+2 l^{*} \gamma$, and $z_{0}=l^{*}(1+\gamma)$.

Incorporating the influence of Brownian motion of scatterers ${ }^{10,14,15}$ and the expression for $F(\tau)$, we can solve the integration in Eq. (2) over $s$ for the temporal autocorrelation function:

$$
\begin{aligned}
G_{1}(\tau) & =C \frac{\sinh \left(z_{0} \sqrt{\left(S_{U}+S_{B}+\mu_{a}\right) D^{-1}}\right)}{\sinh \left(L_{0} \sqrt{\left(S_{U}+S_{B}+\mu_{a}\right) D^{-1}}\right)}, \\
C & =\sinh \left(L_{0} \sqrt{\mu_{a} D^{-1}}\right) / \sinh \left(z_{0} \sqrt{\mu_{a} D^{-1}}\right)
\end{aligned}
$$




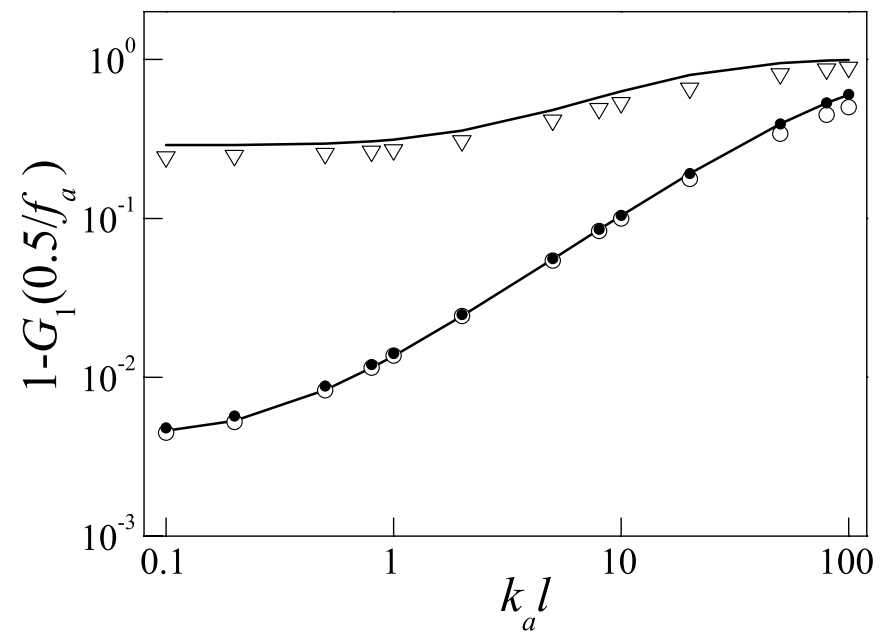

Figure 1. The $k_{a} l$ dependence of the maximum variation of the time autocorrelation function while $k_{a}$ is kept constant. Different lines are for different values of the scattering anisotropy factor $g$ and the acoustic amplitude $A$. Empty symbols indicate the Monte Carlo results: $\bigcirc(g=0.9, A=3.5 \AA), \nabla(g=0, A=3.5 \AA)$. Solid lines indicate the analytical results. Filed symbols indicate the analytical results as well but by using the similarity relation. The following parameters are used in the calculations: $L / l=127.35$, the wavelength of light in vacuo is $\lambda_{0}=500 \mathrm{~nm}, n_{0}=1.33, f_{a}=1 \mathrm{MHz}, v_{a}=1480$ $\mathrm{m} / \mathrm{s}$, and $\eta=0.3211$.

where $S_{B}=2 \tau /\left(\tau_{0} l^{*}\right)$ is the term due to Brownian motion ( $\tau_{0}$ is the single-particle relaxation time), and $S_{U}$ is the term due to the ultrasonic influence:

$$
S_{U}=\frac{1}{2}\left(2 n_{0} k_{0} A\right)^{2} \sin ^{2}\left(\omega_{a} \tau / 2\right)\left(\delta_{n}+\delta_{d}\right)
$$

\section{MONTE CARLO SIMULATION}

To provide an independent numerical approach, we modified the existing public-domain Monte Carlo package ${ }^{17}$ for the transport of light in scattering media to sample the autocorrelation function according to Eqs. (2) and (3). Because it would be very time-consuming to physically simulate a point detector using the Monte Carlo code, we applied the principle of reciprocity in our simulation : the slab is illuminated by a point source and the transmitted light is collected by a plane detector. The scattering angle of a photon in our Monte Carlo simulation is determined by the Henyey-Greenstein phase function, ${ }^{18}$ but it would be trivial to extend it to any analytically or numerically defined phase function. For details of the Monte Carlo implementation, refer to. ${ }^{12}$

As a first comparison between our analytical solution and the Monte Carlo simulation, we neglect both the optical absorption by setting $\mu_{a}$ to zero and the Brownian motion effect by setting $\tau_{0} \rightarrow \infty$. In Eqs. (17) and (18) we see that the value of $G_{1}(\tau)$ oscillates between 1 at $\tau=0$ and the minimum value at $\tau=\pi / \omega_{a}$. The maximum variation of $G_{1}(\tau)$ is compared for different values of $k_{a} l$ while $k_{a}$ and the ratio $L / l$ (the number of mean free paths in a slab of thickness $L$ ) are kept constant. We repeat the test for several different values of the scattering anisotropy factor $g$ and the acoustic amplitude $A$.

The results are shown in Fig. 1. The analytical predictions (solid lines in Fig. 1) fit the Monte Carlo calculations (empty scatterers) very well. In general, increasing the value of $g$ leads to a decreased maximum variation of $G_{1}(\tau)$ due to a decreased number of equivalent isotropic scattering events inside the slab. Further, a larger ultrasonic amplitude increases the maximum variation of the temporal autocorrelation function due to the larger movement of scattering centers and greater modulation of the index of refraction. Finally, the maximum variation grows in a slab geometry with $k_{a} l$ due to the larger value of the product $l \delta_{n}$, while the product $l \delta_{d}$ 

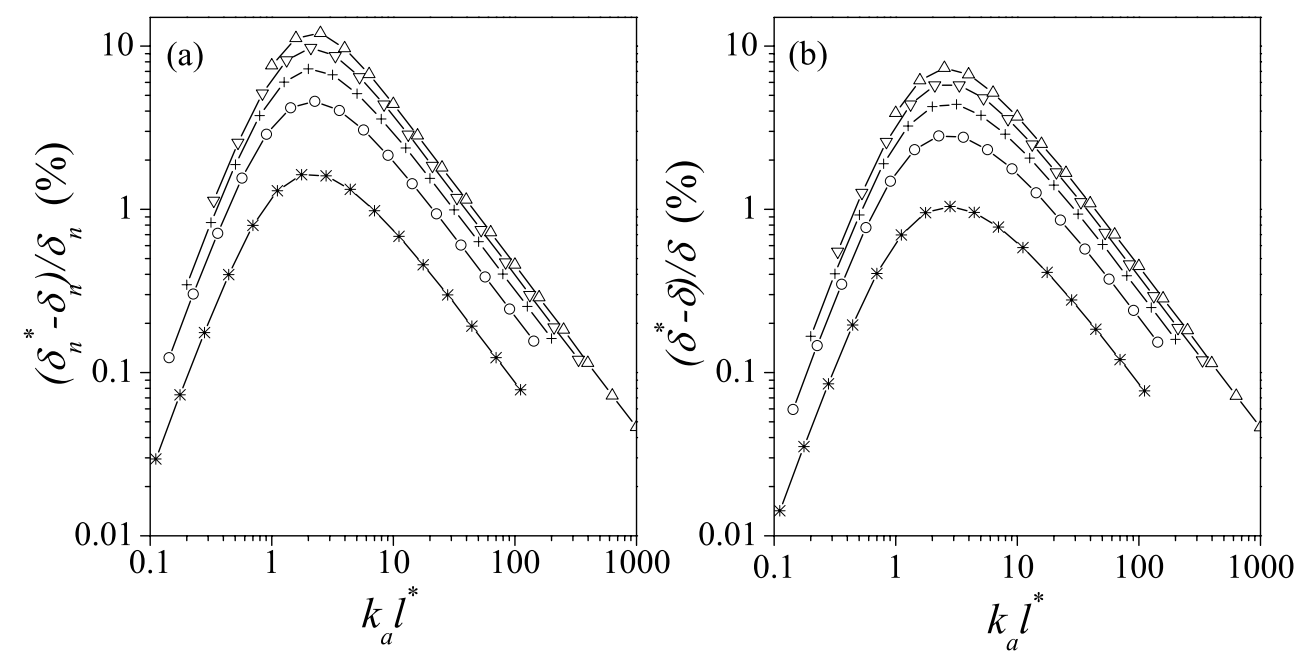

Figure 2. Relative error of (a) $\delta_{n}^{*}$, and (b) $\delta^{*}$ for different $k_{a} l^{*}$ and $g$ values. Lines $(*, \bigcirc,+, \nabla, \triangle)$ represent respectively $(0.1,0.3,0.5,0.7,0.9)$ values of the scattering anisotropy factor $g$.

remains unchanged. From Fig. 1 we see that our analytical model works well for a wide range of $k_{a} l$ even when the anisotropy factor is non-zero.

\section{SIMILARITY RELATION}

In this section, we will explore a similarity relation using the verified analytical solution, rather than the numerical solution shown previously. ${ }^{12}$ In intensity- based photon transport theory, there is a similarity relation ${ }^{19}$ : if the transport scattering coefficient $\mu_{s}^{*}\left[=\mu_{s}(1-g)\right]$ remains constant when the scattering coefficient $\mu_{s}$ and the scattering anisotropy factor $g$ vary, the spatial distribution of light intensity will be approximately the same. The similarity relation $\left[\mu_{s}^{*}=\mu_{s}(1-g)\right]$ can be rewritten as $l^{*}=l /(1-g)$, where $l^{*}$ is the isotropic scattering mean free path. Here, we will examine the counterpart of this conventional similarity relation in the ultrasonic modulation of coherent light. In other words, we will compare two cases: (1) the scattering coefficient is $\mu_{s}$ and the scattering anisotropy factor is $g$ and (2) the scattering coefficient is $\mu_{s}^{*}\left[=\mu_{s}(1-g)\right]$ and the scattering anisotropy factor is zero. In the following text, the symbols with * indicate case (2).

In Eq. (13) we see that the values of $\delta_{d}$ for both the cases are exactly the same $\left(\delta_{d}=\delta_{d}^{*}\right)$. On the other hand, the matrix $\hat{J}$ for the isotropic case (2) reduces to only one number: $\chi=\arctan \left(k_{a} l^{*}\right) /\left(k_{a} l^{*}\right)$ and we have $\delta_{n}^{*}=\eta^{2} k_{a}^{2} l^{*} \chi /(1-\chi) \cdot{ }^{11}$ However, the matrix $\hat{J}$ for the general case (1) is quite complicated, and a direct analytical comparison with case (2) is difficult. Instead, we will plot the relative error between the two cases.

From Fig. 2(a), we see that the discrepancy between $\delta_{n}^{*}$ and $\delta_{n}$ is not very large (less than 13 percent), even when the scattering anisotropy factor $g$ is 0.9 . The error grows with $g$ and has a maximum around $k_{a} l^{*}=2$. Because the $\delta_{d}$ part of the sum $\delta=\delta_{n}+\delta_{d}$ is unchanged by the similarity transformation, the relative difference between $\delta^{*}$ and $\delta$ is even smaller. From Fig. 2(b) we see that the relative error of $\delta^{*}$ is less than 8 percent. The validity of the similarity relation can also been seen in Fig. 1 (Sec. 4).

In conclusion, with a relatively small error, we can apply the similarity relation in the calculation of the temporal autocorrelation function under the conditions we considered during the derivation of $F(\tau)$ and $G_{1}(\tau)$.

\section{CONCLUSION}

In conclusion, we have presented an analytical solution for the autocorrelation function of an ultrasoundmodulated electric field along a path with $N$ scatterers when scattering is anisotropic. A further analytical 
solution was found for light transmitted through a scattering slab using a plane source and a point detector. Using a Monte Carlo simulation, we verified the accuracy of the analytical solution. We also tested the similarity relation and showed that it can be used as a good approximation in the calculation of the autocorrelation function. Our analytical solution is valid under the following conditions: diffusion regime transport, a small ultrasonic modulation, and the value of $k_{a} l$ is not too small.

\section{APPENDIX A.}

The averaging over time of each term on the right side of Eq. (8) and over the lengths $l_{j}$ of all free paths produce:

$$
\begin{aligned}
\left\langle\sum_{j=1}^{N}\left(\Delta \phi_{n, j}(t, \tau)\right)^{2}\right\rangle_{t, l_{j}}= & \frac{1}{8}\left(4 n_{0} k_{0} A \eta\right)^{2} \sin ^{2}\left(\frac{1}{2} \omega_{a} \tau\right)\left(k_{a} l\right)^{2} \sum_{j=1}^{N}\left(T\left(x_{j}\right)+T^{*}\left(x_{j}\right)\right), \\
\left\langle 2 \sum_{j=2}^{N} \sum_{k=1}^{j-1} \Delta \phi_{n, j} \Delta \phi_{n, k}\right\rangle_{t, l_{j}}= & \frac{1}{8}\left(k_{a} l\right)^{2}\left(4 n_{0} k_{0} A \eta\right)^{2} \sin ^{2}\left(\frac{1}{2} \omega_{a} \tau\right) \\
& \times \sum_{j=2}^{N} \sum_{k=1}^{j-1}\left(\prod_{m=k}^{j} T\left(x_{m}\right)+\prod_{m=k}^{j} T^{*}\left(x_{m}\right)\right) \\
\left\langle\sum_{j=1}^{N-1}\left(\Delta \phi_{d, j}(t, \tau)\right)^{2}\right\rangle_{t, l_{j}}= & \frac{1}{2}\left(2 n_{0} k_{0} A\right)^{2} \sin ^{2}\left(\frac{1}{2} \omega_{a} \tau\right) \sum_{j=1}^{N-1}\left(x_{j+1}-x_{j}\right)^{2}, \\
\left\langle 2 \sum_{j=2}^{N-1} \sum_{k=1}^{j-1} \Delta \phi_{d, j} \Delta \phi_{d, k}\right\rangle_{t, l_{j}}= & \frac{1}{2}\left(2 n_{0} k_{0} A\right)^{2} \sin ^{2}\left(\frac{1}{2} \omega_{a} \tau\right) \sum_{j=2}^{N-1} \sum_{k=1}^{j-1}\left\{\left[\left(\hat{\mathbf{e}}_{j+1}-\hat{\mathbf{e}}_{j}\right) \cdot \hat{\mathbf{e}}_{a}\right]\right. \\
\left\langle 2 \sum_{j=1}^{N} \sum_{k=1}^{N-1} \Delta \phi_{n, j} \Delta \phi_{d, k}\right\rangle_{t, l_{j}}= & \frac{1}{2} k_{a} l\left(2 n_{0} k_{0} A\right)^{2} \eta \sin ^{2}\left(\frac{1}{2} \omega_{a} \tau\right) \\
& \left.\times\left[\left(\hat{\mathbf{e}}_{k+1}-\hat{\mathbf{e}}_{k}\right) \cdot \hat{\mathbf{e}}_{a}\right]\left(\prod_{m=k+1}^{j} T\left(x_{m}\right)+\prod_{m=k+1}^{j} T^{*}\left(x_{m}\right)\right)\right\}, \\
& +\sum_{j=2}^{N-1} \sum_{k=1}^{N-1} \sum_{k=j}^{N-1}\left(x_{k+1}-x_{k}\right)\left(\prod_{m=j}^{k} T\left(x_{m}\right)+\prod_{m=j}^{k} T^{*}\left(x_{m}\right)\right) \\
& \left.\times \prod_{m=k}^{j} T_{m=k+1}^{j} T\left(x_{m}\right)+\prod_{m}^{*}\left(x_{m}\right)\right),
\end{aligned}
$$

where $T\left(x_{m}\right)=1 /\left(1-i k_{a} l x_{m}\right), T^{*}\left(x_{m}\right)$ is its complex conjugate, $i$ is imaginary unit, and we use a variable $x_{m}$ to represent $\cos \theta_{m}$.

In order to provide averaging over all scattering directions, as a first step we expand the phase function for the polar angle $f(\cos \theta)$ over Legendre polynomials:

$$
f(\cos \theta)=\sum_{m=0}^{\infty} \frac{2 m+1}{2} g_{m} P_{m}(\cos \theta), \quad g_{m}=\int_{0}^{\pi} f(\cos \theta) P_{m}(\cos \theta) \sin \theta d \theta
$$

where $\cos \theta$ represents the cosine of the deflection angle.

Notice that in Eq. (24) $g_{0}=1$, and $g_{1}$ is equal to the scattering anisotropy factor $g$. In the case of HenyeyGreenstein phase function for the polar angle, ${ }^{18}$ the value of each coefficient $g_{m}$ is the $m$ th power of the scattering 
anisotropy factor $\left(g_{m}=g^{m}\right)$. Because the azimuth angles are uniformly distributed, the phase function for both the azimuth and polar angles are simply the polar phase function multiplied by a constant factor $(2 \pi)^{-1}$.

In our case the argument of the phase function is the cosine of the angle between the incoming and outgoing photon direction $\left(\hat{\mathbf{e}}_{j} \cdot \hat{\mathbf{e}}_{j+1}\right)$. The unity vector $\hat{\mathbf{e}}_{j}$ in a spherical coordinate system has a form $\hat{\mathbf{e}}_{j}=\cos \theta_{j} \hat{\mathbf{e}}_{a}+$ $\sin \theta_{j} \cos \varphi_{j} \hat{\mathbf{e}}_{x}+\sin \theta_{j} \sin \varphi_{j} \hat{\mathbf{e}}_{y}$, and the argument of the phase function in this representation becomes

$$
\cos \theta=\cos \theta_{j} \cos \theta_{j+1}+\sin \theta_{j} \sin \theta_{j+1} \cos \left(\varphi_{j}-\varphi_{j+1}\right) .
$$

Using the identity ${ }^{20}$

$$
\begin{aligned}
P_{n}\left(x y-\sqrt{1-x^{2}} \sqrt{1-y^{2}} \cos (\alpha)\right)= & P_{n}(x) P_{n}(y) \\
& +2 \sum_{k=1}^{n} \frac{(-1)^{k} \cos (k \alpha)(n-k) !}{(k+n) !} P_{n}^{k}(x) P_{n}^{k}(y),
\end{aligned}
$$

and representing $x, y$, and $\alpha$ with $\cos \theta_{j}, \cos \theta_{j+1}$, and $\pi+\varphi_{j}-\varphi_{j+1}$, we first provide integration over all uniformly distributed azimuth angles in Eqs. (19-23). Because in Eqs. (19-23) nothing depends on the azimuth angle, all terms with Associate Legendre polynomials $P_{n}^{k}()$ in Eq. (26) vanish during the integration. Thus, for the further integration over the polar angles, the probability density function of the photon to travel along the directions $\hat{\mathbf{e}}_{1}, \ldots, \hat{\mathbf{e}}_{N}$ reduce to the function $f^{(N)}\left(\cos \theta_{1}, \ldots, \cos \theta_{N}\right)$, which depends only on the polar angles along the photon path:

$$
\begin{aligned}
f^{(N)}\left(\cos \theta_{1}, \ldots, \cos \theta_{N}\right) & =\tilde{p}_{s}\left(\cos \theta_{1}\right) \prod_{j=1}^{N-1} f^{(2)}\left(\cos \theta_{j}, \cos \theta_{j+1}\right), \\
f^{(2)}\left(\cos \theta_{j}, \cos \theta_{j+1}\right) & =\sum_{m=0}^{\infty} \frac{2 m+1}{2} g_{m} P_{m}\left(\cos \theta_{j}\right) P_{m}\left(\cos \theta_{j+1}\right)
\end{aligned}
$$

where $\tilde{p}_{s}\left(\cos \theta_{1}\right)$ is the probability density function of the starting polar angle. For simplicity, we assume $\tilde{p}_{s}\left(\cos \theta_{1}\right)=1 / 2$ (uniform distribution) instead of the actual anisotropic phase function, which was shown not to affect the final result in the diffusion regime.

Using the orthogonality of Legendre polynomials, now it is straightforward to obtain the following equations:

$$
\begin{aligned}
H_{j}\left(x_{j-1}, x_{j+1}\right) & =\int_{-1}^{1} f^{(2)}\left(x_{j-1}, x_{j}\right) T\left(x_{j}\right) f^{(2)}\left(x_{j}, x_{j+1}\right) d x_{j} \\
& =\sum_{m=0}^{\infty} \sum_{n=0}^{\infty} g_{m}^{1 / 2} g_{n}^{1 / 2} \sqrt{\frac{2 m+1}{2}} \sqrt{\frac{2 n+1}{2}} J_{m, n} P_{m}\left(x_{j-1}\right) P_{n}\left(x_{j+1}\right), \\
\left\langle T\left(x_{j}\right)\right\rangle_{x_{i}} & =\int_{-1}^{1} \ldots \int_{-1}^{1} T\left(x_{j}\right) f^{(N)}\left(x_{1}, \ldots, x_{N}\right) d x_{1} \ldots d x_{N} \\
& =(\hat{J})_{0,0}, \\
\left\langle\prod_{m=k}^{j} T\left(x_{m}\right)\right\rangle_{x_{i}} & =\int_{-1}^{1} \ldots \int_{-1}^{1}\left(\prod_{m=k}^{j} T\left(x_{m}\right)\right) f^{N}\left(x_{1}, \ldots, x_{N}\right) d x_{1} \ldots d x_{N}
\end{aligned}
$$




$$
\begin{aligned}
& =\sum_{i(1)=0}^{\infty} \ldots \sum_{i(j-k)=0}^{\infty} J_{0, i(1)} J_{i(1), i(2)} \ldots J_{i(j-k), 0} \\
& =\left(\hat{J}^{j-k+1}\right)_{0,0},
\end{aligned}
$$

where $\hat{J}$ is the matrix defined by

$$
J_{m, n}=g_{m}^{1 / 2} g_{n}^{1 / 2} \sqrt{\frac{2 m+1}{2}} \sqrt{\frac{2 n+1}{2}} \int_{-1}^{1} T(x) P_{m}(x) P_{n}(x) d x
$$

and the $(\hat{J})_{0,0}$ represents the $(0,0)$ element of the matrix $\hat{J}$.

Thus, the average of the right side of Eq. (19) over all the polar angles becomes

$$
\left\langle\sum_{j=1}^{N}\left(\Delta \phi_{n, j}(t, \tau)\right)^{2}\right\rangle_{t, l_{j}, x_{i}}=\frac{1}{8}\left(4 n_{0} k_{0} A \eta\right)^{2} \sin ^{2}\left(\frac{1}{2} \omega_{a} \tau\right)\left(k_{a} l\right)^{2} N\left[(\hat{J})_{0,0}+\left(\hat{J}^{*}\right)_{0,0}\right] .
$$

On the other hand, the average of the right side of Eq. (20) has a more complicated form:

$$
\begin{aligned}
\left\langle 2 \sum_{j=2}^{N} \sum_{k=1}^{j-1} \Delta \phi_{n, j} \Delta \phi_{n, k}\right\rangle_{t, l_{j}, x_{i}}= & \frac{1}{8}\left(k_{a} l\right)^{2}\left(4 n_{0} k_{0} A \eta\right)^{2} \sin ^{2}\left(\frac{1}{2} \omega_{a} \tau\right) \\
& \times \sum_{j=2}^{N} \sum_{k=1}^{j-1}\left[\left(\hat{J}^{j-k+1}\right)_{0,0}+\left(\hat{J}^{j-k+1}\right)_{0,0}\right] .
\end{aligned}
$$

If we replace the sums on the right side of Eq. (34) with

$$
\sum_{j=2}^{N} \sum_{k=1}^{j-1}\left(\hat{J}^{j-k+1}\right)_{0,0}=\left\{\hat{J}^{2}(\hat{I}-\hat{J})^{-1}\left[(N-1) \hat{I}-\hat{J}\left(\hat{I}-\hat{J}^{N-1}\right)(\hat{I}-\hat{J})^{-1}\right]\right\}_{0,0},
$$

and further keep only the terms that are proportional to a large number $N$ in the above equation, we have

$$
\begin{aligned}
\left\langle 2 \sum_{j=2}^{N} \sum_{k=1}^{j-1} \Delta \phi_{n, j} \Delta \phi_{n, k}\right\rangle_{t, l_{j}, x_{i}} \simeq & N \frac{1}{8}\left(k_{a} l\right)^{2}\left(4 n_{0} k_{0} A \eta\right)^{2} \sin ^{2}\left(\frac{1}{2} \omega_{a} \tau\right) \\
& \times\left[\hat{J}^{2}(\hat{I}-\hat{J})^{-1}+\hat{J}^{*}\left(\hat{I}-\hat{J}^{*}\right)^{-1}\right]_{0,0} .
\end{aligned}
$$

Joining equations (33) and (36), we finally have

$$
\begin{aligned}
\left\langle\sum_{j=1}^{N}\left(\Delta \phi_{n, j}(t, \tau)\right)^{2}+2 \sum_{j=2}^{N} \sum_{k=1}^{j-1} \Delta \phi_{n, j} \Delta \phi_{n, k}\right\rangle_{t, l_{j}, x_{i}} & \simeq \frac{1}{4}\left(k_{a} l\right)^{2}\left(4 n_{0} k_{0} A \eta\right)^{2} \sin ^{2}\left(\frac{1}{2} \omega_{a} \tau\right) \\
& \times \Re\left[\hat{J}(\hat{I}-\hat{J})^{-1}\right]_{0,0},
\end{aligned}
$$


where $\Re$ is for the real value.

The remaining task is to provide the average of the right side of Eqs. (21), (22), and (23), over all polar angles. As a first step, we define the coefficient $\Phi_{m, n}$ for any function $\Phi(x)$, and for each pair of nonnegative integer numbers $(m, n)$ :

$$
\Phi_{m, n}=\int_{-1}^{1} \sqrt{\frac{2 m+1}{2}} \sqrt{\frac{2 n+1}{2}} g_{m}^{1 / 2} g_{n}^{1 / 2} \Phi(x) P_{m}(x) P_{n}(x) d x .
$$

Then, according to the definition in Eq. (38), it is easy to show that for the functions $x, x^{2}, T(x)$, and $x T(x)$ we have

$$
\begin{aligned}
(x)_{0, j} & =\delta_{1, j} \sqrt{g_{1}} / 3 \\
\left(x^{2}\right)_{0,0} & =1 / 3 \\
(x T(x))_{0, j} & =\left(i k_{a} l\right)^{-1}\left(T_{0, j}-\delta_{0, j}\right) \\
T_{j, 1} & =\left(i k_{a} l\right)^{-1} \sqrt{3 g_{1}}\left(T_{0, j}-\delta_{0, j}\right),
\end{aligned}
$$

where $\delta_{a, b}$ represents the delta function.

Using the results in Eq. (39), the average over all the polar angles of the right side of Eq. (21) becomes

$$
\left\langle\sum_{j=1}^{N-1}\left(\Delta \phi_{d, j}(t, \tau)\right)^{2}\right\rangle_{t, l_{j}, x_{i}}=\frac{1}{2}\left(2 n_{0} k_{0} A\right)^{2} \sin ^{2}\left(\frac{1}{2} \omega_{a} \tau\right)(N-1) \frac{2}{3}\left(1-g_{1}\right) .
$$

On the other hand, the average of the right side of Eq. (22) is

$$
\begin{aligned}
\left\langle 2 \sum_{j=2}^{N-1} \sum_{k=1}^{j-1} \Delta \phi_{d, j} \Delta \phi_{d, k}\right\rangle_{t, l_{j}, x_{i}}= & \left(2 n_{0} k_{0} A\right)^{2} \sin ^{2}\left(\frac{1}{2} \omega_{a} \tau\right) \\
& \times(1-g)^{2}\left(k_{a} l\right)^{-2} \Re[\hat{M}]_{0,0},
\end{aligned}
$$

where $\hat{M}=\hat{J}^{N-2}-\hat{I}$. Since the right side of Eq. (41) is not proportional to $N$, we consider it much smaller than the right side of Eq. (40), and we have

$$
\left\langle\sum_{j=1}^{N-1}\left(\Delta \phi_{d, j}(t, \tau)\right)^{2}+2 \sum_{j=2}^{N-1} \sum_{k=1}^{j-1} \Delta \phi_{d, j} \Delta \phi_{d, k}\right\rangle_{t, l_{j}, x_{i}} \simeq N \frac{1}{2}\left(2 n_{0} k_{0} A\right)^{2} \sin ^{2}\left(\frac{1}{2} \omega_{a} \tau\right) \frac{2}{3}\left(1-g_{1}\right) .
$$

In general, the errors of approximation we made in Eqs. (37) and (43) are small when both $k_{a} l$ and the average $N$ are large. Conversely, the error can be large: for example, if $N=10$, and $k_{a} l=1$, the error is about $50 \%$ for isotropic scattering.

Finally, the average over all the polar angles of the right side of Eq. (23) is

$$
\begin{aligned}
\left\langle 2 \sum_{j=1}^{N} \sum_{k=1}^{N-1} \Delta \phi_{n, j} \Delta \phi_{d, k}\right\rangle_{t, l_{j}, x_{i}} & =\left(1-g_{1}\right)\left(k_{a} l\right)^{-1} i(N-1)\left[\left(\hat{J}^{2}\right)_{0,0}-\left(\hat{J}^{*}\right)_{0,0}\right] \\
& =0 .
\end{aligned}
$$


The zero is obtained in Eq. (44) because the elements of the symmetric matrix $\hat{J}$ are either real or imaginary numbers.

The expression for the function $F(\tau)$ [Eq. (4)] becomes

$$
\begin{aligned}
F(\tau) \simeq & \left\langle N \frac{1}{4}\left(k_{a} l\right)^{2}\left(4 n_{0} k_{0} A \eta\right)^{2} \sin ^{2}\left(\frac{1}{2} \omega_{a} \tau\right) \Re\left[\hat{J}(\hat{I}-\hat{J})^{-1}\right]_{0,0}\right. \\
& \left.+N \frac{1}{2}\left(2 n_{0} k_{0} A\right)^{2} \sin ^{2}\left(\frac{1}{2} \omega_{a} \tau\right) \frac{2}{3}\left(1-g_{1}\right)\right\rangle_{N},
\end{aligned}
$$

where the last average is over all realizations of the number of free paths $N$ in a photon path of length $s$. Since the average value of $N$ is $s / l$, we have

$$
F(\tau) \simeq \frac{s}{l}\left(2 n_{0} k_{0} A\right)^{2} \sin ^{2}\left(\frac{1}{2} \omega_{a} \tau\right)\left\{\eta^{2}\left(k_{a} l\right)^{2} \Re\left[\hat{J}(\hat{I}-\hat{J})^{-1}\right]_{0,0}+\frac{1}{3}\left(1-g_{1}\right)\right\}
$$

\section{REFERENCES}

1. F. A. Marks, H. W. Tomlinson, and G. W. Brooksby, "A comprehensive approach to brest cancer detection using light: photon localization by ultrasound modulation and tissue characterization by spectral discrimination," in Proc. Soc. Photo-Opt. Instrum. Eng., 1888, pp. 500-510, 1993.

2. G. D. Mahan, W. E. Engler, J. J. Tiemann, and E. G. Uzgiris, "Ultrasonic tagging of light: Theory," in Proc. Natl. Acad. Sci. USA, 95, p. 14015, 1998.

3. L.-H. V. Wang, S. L. Jacques, and X. Zhao, "Continuous-wave ultrasonic modulation of scattered laser light to image objects in turbid media," Opt. Lett. 20, pp. 629-631, 1995.

4. M. Kempe, M. Larionov, D. Zaslavsky, and A. Z. Genack, "Acousto-optic tomography with multiply scattered light," J. Opt. Soc. Am. A 14, pp. 1151-1158, 1997.

5. L.-H. V. Wang and G. Ku, "Frequency-swept ultrasound-modulated optical tomography of scattering media," Opt. Lett. 23, pp. 975-977, 1998.

6. S. Leveque, A. C. Boccara, M. Lebec, and H. Saint-Jalmes, "Ultrasonic tagging of photon paths in scattering media: parallel speckle modulation processing," Opt. Lett. 24, pp. 181-183, 1999.

7. G. Yao and L.-H. V. Wang, "Theoretical and experimental studies of ultrasound-modualted optical tomography in biological tissue," Appl. Opt. 39, pp. 659-664, 2000.

8. G. Yao, S.-L. Jiao, and L.-H. V. Wang, "Frequency-swept ultrasound-modulated optical tomography in biological tissue by use of parallel detection," Opt. Lett. 25, pp. 734-736, 2000.

9. A. Lev, Z. Kotler, and B. G. Sfez, "Ultrasound tagged light imaging in turbid media in a reflectance geometry," Opt. Lett. 25, pp. 378-380, 2000.

10. W. Leutz and G. Maret, "Ultrasonic modulation of multiply scattered-light," Physica B 204, pp. 14-19, 1995.

11. L.-H. V. Wang, "Mechanisms of ultrasonic modulation of multiply scattered coherent light: an analytic model," Phys. Rev. Lett. 87, pp. 043903-(1-4), 2001.

12. L.-H. V. Wang, "Mechanisms of ultrasonic modulation of multiply scattered coherent light: a monte carlo model," Opt. Lett. 26, pp. 1191-1193, 2001.

13. M. S. Patterson, B. Chance, and B. C. Willson, "Time resolved reflectance and transmittance for the noninvasive measurement of tissue optical-properties," Appl. Opt. 28, pp. 2331-2336, 1989.

14. G. Maret and P. E. Wolf, "Multiple light-scattering from disordered media - the effect of brownian-motion of scatterers," Z. Phys. B 65, pp. 409-413, 1987.

15. D. J. Pine, D. A. Weitz, P. M. Chaikin, and E. Herbolzheimer, "Diffusing-wave spectroscopy," Phys. Rev. Lett. 60, pp. 1134-1137, 1988.

16. A. Ishimaru, Wave Propagation and Scattering in Random Media, Academic, New York, 1978. 
17. L.-H. V. Wang, S. L. Jacques, and L.-Q. Zheng, "Mcml - monte carlo modeling of photon transport in multi-layered tissues," Comput. Methods Prog. Biomed. 47, pp. 131-146, 1995.

18. L. G. Henyey and J. L. Greenstein, "Diffuse radiation in the galaxy," Astrophys. J. 93, pp. 70-83, 1941.

19. D. R. Wyman, M. S. Patterson, and B. C. Wilson, "Similarity relations for the interaction parameters in radiation transport," Appl. Opt. 28, pp. 5243-5249, 1989.

20. http://functions.wolfram.com/05.03.06.0022.01 . 